Article

\title{
Entropy Flow and the Evolution of a Storm
}

\section{Ying Liu and Chongjian Liu *}

State Key Laboratory of Severe Weather, Chinese Academy of Meteorological Sciences, Beijing 100081, P. R. China. E-mail: y119@cams.cma.gov.cn

* Author to whom correspondence should be addressed; E-mail: cliu@cams.cma.gov.cn

Received: 5 August 2008; in revised form: 27 September 2008 / Accepted: 27 September 2008 /

Published: 13 October 2008

\begin{abstract}
The universal principle that an open system can be driven to a state far from equilibrium, or organized, by strong negative entropy flow from its surroundings has been validated in numerous fields from physics and chemistry to the life science. In this paper, entropy flows for a severe storm are calculated via the entropy flow formula using the National Centers for Environmental Prediction/National Center for Atmospheric Research reanalysis data. The results show that the intensification of negative (positive) entropy flow entering into the storm preceded the strengthening (weakening) of its intensity, implying that entropy flow analysis can be used as a potential tool in forecasting changes in the intensity of a storm.
\end{abstract}

Keywords: Entropy flow, Storm, Forecast

\section{Introduction}

In recent years more attention has been paid to thermodynamics and statistical mechanics in the atmospheric sciences and other fields studying many-body systems like oceanography. In addition to the large number of journal articles [1-10], several monographs have been published in succession [11-13].

Early in the 1940s Eady pointed out the rather formidable task facing theoretical meteorology - that of discovering the nature of and determining quantitatively all the forecastable regularities of a "permanently unstable" (i.e., permanently turbulent) system, and firmly believed that "we can be certain that these regularities are necessarily statistical and to this extent our technique must resemble statistical mechanics" [14]. 
Entropy flow is a core concept in non-equilibrium thermodynamics just as entropy is in classical thermodynamics and statistical physics. According to the second law of thermodynamics, an isolated system will evolve spontaneously into the equilibrium with maximum entropy in which the order of the system is a minimum [15-16]. As a result, negative entropy is very important for an open system to remain far from equilibrium, which is also true in biological systems as reflected in the statement that life's existence depends on its continuous gain of "negentropy" from its surroundings [17-18].

The atmosphere has been likened to a giant thermodynamic engine in which disorganized heat energy is transformed into the organized kinetic energy of the winds. The general circulation of the atmosphere can be regarded as simply being driven by temperature differences between the polar and equatorial regions [19].

Figure 1. The schematic diagram for Bénard convection as the prototype of selforganization, showing that the temperature difference $\left(T_{2}-T_{1}\right)$ reaching a certain critical value (threshold) will cause the macro-scale convection to occur within the system. Note that the temperature difference $\left(T_{2}-T_{1}\right)>0$ will lead the entropy exchange $\delta s_{e}<0$, meaning that the system gains "negative entropy". See text for details.

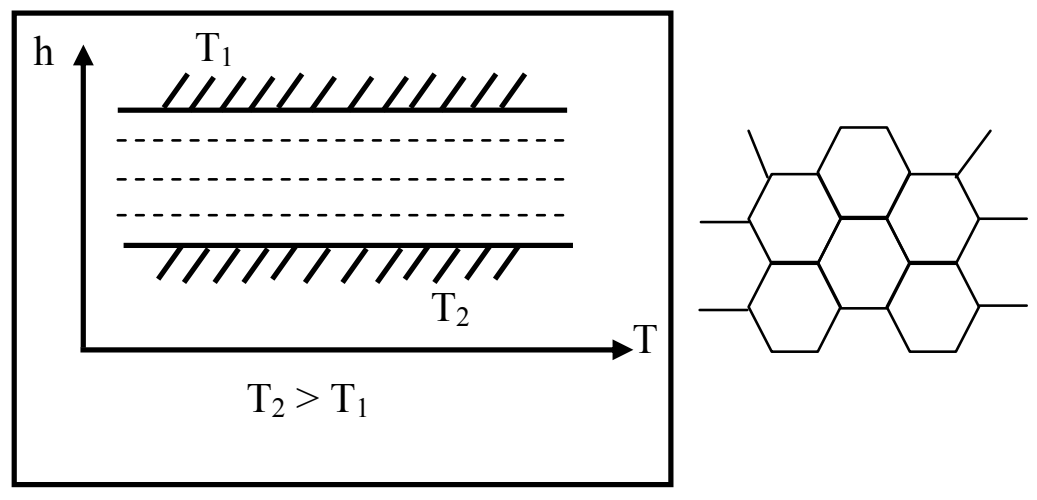

The classical Bénard convective system is driven by a vertical temperature difference, and wellorganized hexagonal convective cells abruptly form within a thin layer of fluid originally at rest once the temperature difference reaches a certain critical value (see Figure 1). This system demonstrates that systems which get heat at a higher temperature and lose heat at a lower temperature will gain "negentropy" from its surroundings. The troposphere experiences a double negative entropy flow process, in the horizontal and vertical, since it gets heat at the tropics and the surface with higher temperature and loses heat at the poles and the tropopause which have lower temperatures, respectively. This suggests a possible explanation for the variety of weather in the troposphere.

In this paper, our starting point is based on the argument that negative entropy flow might drive a system to a state further apart from equilibrium in which its entropy reaches the maximum. It follows that negative entropy flow is important in the sense that it will favor the development or organization of a system such as storm.

In the field of the atmospheric sciences the theory of entropy has been mostly applied to globalscale and/or climatic investigations using the general circulation models or climatic models. For example, the entropy budget of the atmosphere as a whole has been estimated through the entropy 
balance equations and, the global climate system has been examined using the principle of minimum entropy exchange [20-22]. A number of more recent climate studies involve also entropy-flux and entropy production [23-25]. In addition to global-scale researches, a cloud ensemble model is used to study the entropy budget as well [26-27].

With respect to applications of entropy theory to numerical prediction, we would like to mention again the research in which the authors reviewed atmospheric systems in light of non-linear nonequilibrium thermodynamics and reached a series of results, including: numerical simulation of atmospheric circulation improved dramatically when the second law of thermodynamics is incorporated into a global spectrum model [10]; and, the forecast accuracy of a meso-scale numerical weather prediction model is noticeably enhanced by introducing a physics-based diffusion scheme [6-7]. Besides, precursors of cyclogenesis over a specific region have been investigated using the spatial distribution of the moist entropy although the physical connotation of this entropy in their context should be, in nature, different from that of the classical thermodynamic entropy [28].

The purpose of this paper is to derive entropy flow equations for calculating the instantaneous entropy flow field and to discuss the relationship of entropy flow with the life cycle of an atmospheric system - a tropical storm (TS) - with the aim of revealing the dependence of the growth of the system on negative entropy flow. The TS selected for this study is tropical storm Bilis, hereafter called TS Bilis, which struck land on Fujian, China in 2006 and was the worst such disaster in Eastern China in the last ten years, as it caused the most deaths. The entropy flow computations for the various stages of its life-cycle are based on the National Centers for Environmental Prediction/National Center for Atmospheric Research (NCEP/NCAR) $1^{\circ} \times 1^{\circ}$ (latitude-longitude) resolution reanalysis data [29].

\subsection{Background}

The second law of thermodynamics can be expressed in terms of the entropy balance equation provided local equilibrium is assumed [16]. The entropy change in an open system usually consists of two parts: one is entropy flow that is caused by the exchange of entropy in the system with its surroundings and the values of entropy flow can be either negative or positive; and, the other is positive definite entropy production caused by irreversible processes within the system.

The Gibbs relation under the assumption of local equilibrium [30] can be written in terms of the change of entropy per unit mass, s, with time

$$
\frac{d s}{d t}=\frac{1}{T} \frac{d U}{d t}+\frac{p}{T} \frac{d \alpha}{d t}-\sum_{k} \frac{\mu_{k}}{T} \frac{d N_{k}}{d t}
$$

where

$$
N_{k}=\rho_{k} / \rho
$$

and

$$
\rho=\sum_{k} \rho_{k}
$$

The three individual derivatives in the right-hand side of equation 1 can be found through the first law of thermodynamics, the continuity equation and the component balance equation. The first law can be written as 


$$
\frac{d U}{d t}=-\alpha \operatorname{div} \vec{J}_{q}-p \frac{d a}{d t}
$$

where $\alpha=\rho^{-1}$ and $\frac{d \alpha}{d t}$ can be related to $d i v \vec{V}$ via the continuity equation

$$
\frac{d a}{d t}=a d i v \vec{V}
$$

where

$$
\vec{V}=\sum \rho_{k} \bar{V}_{K} / \rho
$$

and, the component balance equation can be written as

$$
\frac{d N_{k}}{d t}=-\alpha d i v J_{k}+\alpha \sum_{r} v_{k r} m_{k} \omega_{r}
$$

Substituting for the three individual time derivatives in the right-hand side of equation 1 by equations 4, 5 and 7 and turning the derivation of entropy into the local derivative, we have

$$
\frac{\partial \rho s}{\partial t}=-\operatorname{div}\left(\rho s \vec{V}+\frac{1}{T} \vec{J}_{q}-\sum_{k} \frac{\mu_{k}}{T} \vec{J}_{k}\right)+\sigma
$$

where

$$
\sigma=-\sum_{k} \vec{J}_{k} \cdot \nabla \frac{\mu_{k}}{T}+\frac{1}{T} \sum_{k} \vec{J}_{k} \cdot \vec{F}_{k}-\frac{1}{T} \sum_{r} \sum_{k} v_{k r} \mu_{k} \omega_{r}
$$

is called entropy production while the "div" term is the entropy flow.

Usually the contribution of $\frac{1}{T} \vec{J}_{q}$ to the entropy flow in the atmosphere is neglectable compared with $\rho s \vec{V}$ while the diffusive flow $\vec{J}_{k}$ for component $k$ can be defined as [31]

$$
\vec{J}_{k}=\rho_{k}\left(\vec{V}_{k}-\vec{V}\right)
$$

In this paper the component of liquid water has been omitted owing to the liquid water content (about $5 \mathrm{gm}^{-3}$ on the average over the tropics) being much less than the density of either vapor or dry air [3233] and only two components are therefore taken into account, that is, vapor and dry air. Usually the velocity $\vec{V}_{q}$ for vapor is assumed to be the same as $\vec{V}_{d}$ for dry air so that $\vec{J}_{k}=0$ in this case. As a consequence, the entropy $s$ per unit mass consists accordingly of $s_{q}$ for vapor and $s_{d}$ for dry air with all the corresponding entropy constants, $s_{q 0}$ and $s_{d 0}$, to vapor and dry air, respectively, set to zero so that

$$
s=s_{q}+s_{d}=q\left(C_{p v} \ln T-R_{v} \ln e\right)+(1-q)\left[C_{p d} \ln T-R_{d} \ln (p-e)\right]
$$

The formulas above are derived based on Cartesian coordinates but the NCEP/NCAR reanalysis data used in this paper are on constant pressure layers, so it is necessary to transform the expression of entropy flow into that in the p-coordinates

$$
-d i v \vec{J}_{s}=-\frac{\partial \rho s u}{\partial x}-\frac{\partial \rho s v}{\partial y}+\rho g \frac{\partial \rho s \omega}{\partial p}
$$

Equation 12 is the fundamental formula for diagnosing entropy flow employed in this paper.

\subsection{Case Description}

TS Bilis struck land at Fujian, China on 14 July 2006, and caused direct economic losses of US\$5.1 billion, 654 deaths and 208 missing persons. TS Bilis formed in the afternoon on 9 July 2006, 
developed and reached maturity on 0000 UTC 12 July. Its prime was from 0000 UTC 13 July to 0000 UTC 14 July, after which it began to decay.

The entropy flows for the different evolutionary stages of this storm are examined. These flows are evaluated using equation 12 and the NCEP/NCAR $1^{\circ} \times 1^{\circ}$ (latitude-longitude) resolution reanalysis data [29].

\section{Results and Discussion}

Changes in the entropy flow at the near surface $850 \mathrm{hPa}$ layer are examined initially and related to the evolution of TS Bilis. A more detailed discussion about the dependence of the growth of the threedimensional storm as a thermodynamic system on the negative entropy flow for its whole life-cycle is made in the last portion of this paper.

Figure 2. Entropy flow fields at $850 \mathrm{hPa}$ in the different evolution stages of TS Bilis (2006): (a) genesis (0600 UTC 10 July), (b) development (0000 UTC 12 July), (c) maturity (0000 UTC 13 July), and (d) decay (0000 UTC 14 July). The contour interval is $0.5 \times 10^{3}$ $\mathrm{JK}^{-1} \mathrm{~s}^{-1}$, and the circle is centered on the storm and indicates its extent.
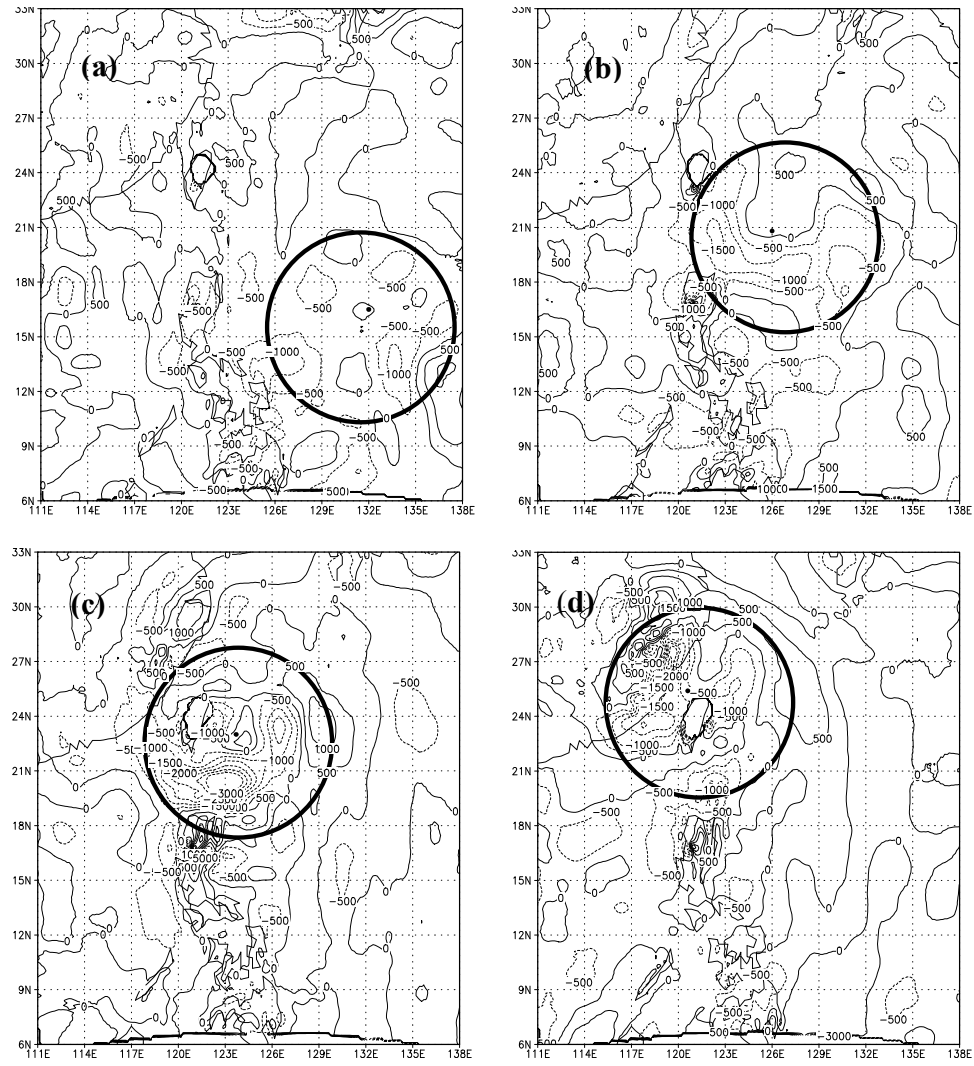

Representative times for TS Bilis genesis or its initial stage, development, maturity and decay stages are determined as 0600 UTC 10, 0000 UTC 12, 0000 UTC 13 and 0000 UTC 14 July, respectively.

In the initial stage [Figure 2(a)], negative entropy flow dominates the circled region, with a maximum negative entropy flow of about $-1 \times 10^{3} \mathrm{JK}^{-1} \mathrm{~s}^{-1}$. TS Bilis develops with time and the area with negative entropy flow greater than $-1 \times 10^{3} \mathrm{JK}^{-1} \mathrm{~s}^{-1}$ within the region expands with the maximum 
negative entropy flow strengthening to around $-1.5 \times 10^{3} \mathrm{JK}^{-1} \mathrm{~s}^{-1}$ as seen at 0000 UTC 12 July [Figure 2(b)]. TS Bilis then enters its mature stage with a central pressure below $980 \mathrm{hPa}$ until 0000 UTC 13 July when the negative entropy flow around the storm center and in its neighborhood reaches a maximum negative entropy flow greater than $-3 \times 10^{3} \mathrm{JK}^{-1} \mathrm{~s}^{-1}$ [Figure 2 (c)]. From 0600 UTC 13 to 0000 UTC 14 July TS Bilis is at its maximum with a central pressure down to $975 \mathrm{hPa}$. At $0000 \mathrm{UTC} 14$ July it starts to decay and the corresponding negative entropy flow is decreased [Figure 2 (d)].

Next the longitude- and latitude-vertical cross sections of entropy flow through the storm center will be analyzed in order to further understand the dependence of the growth of the storm on negative entropy flow.

Figure 3. The pressure-longitudinal (a)/(c) and pressure-latitudinal (b)/(d) cross sections of entropy flow through TS Bilis' centre at 0600 UTC 10/0000 UTC 13 July, respectively. The contour interval is $0.5 \times 10^{3} \mathrm{~J} \mathrm{~K}^{-1} \mathrm{~s}^{-1}$, and the black triangles indicate the respective positions of the storm center at the surface.
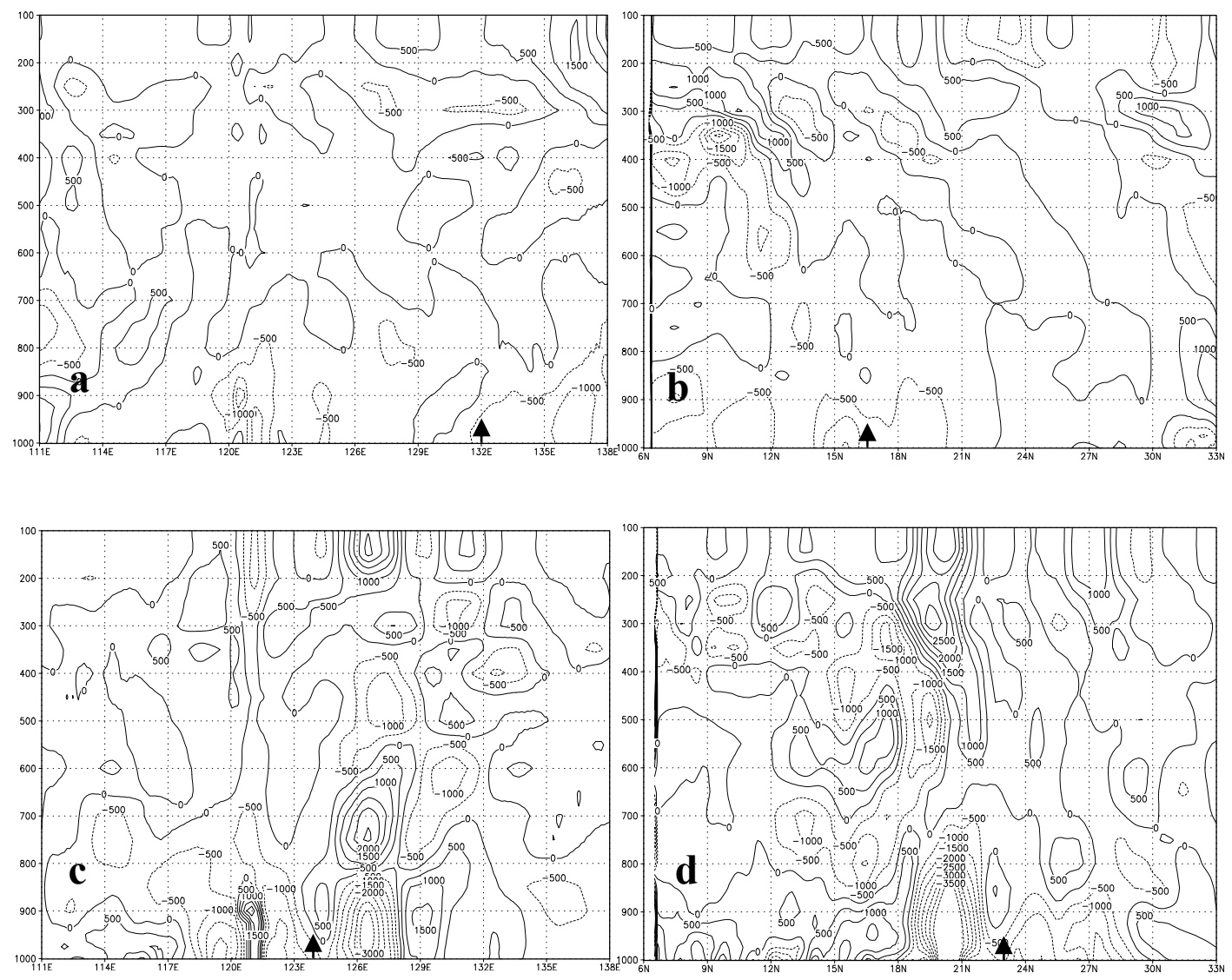

Figure 3 shows the cross sections of entropy flow at 0600 UTC 10 and 0000 UTC 13 July for the genesis and mature stages of TS Bilis, respectively.

During the genesis stage negative entropy flow is weak, especially in the lower troposphere [Figures 3(a)-3(b)]. It gradually becomes stronger and expands toward the upper levels with the rapid development of the storm. In the development stage areas of negative entropy flow have reached 300 $h P a$ in the longitudinal cross section, and in the mature stage these regions have almost penetrated the whole troposphere up to $200 \mathrm{hPa}$ or higher [Figures 3(c)-3(d)]. Such vertical penetration of negative 
entropy flow continues until 0000 UTC 14 July when TS Bilis starts to decay with a drop of $5 \mathrm{~ms}^{-1}$ in the maximum surface wind and rise by $5 \mathrm{hPa}$ in the central pressure within $6 \mathrm{~h}$. This suggests that changes in the entropy flow should be an indication of changes in the intensity of a storm.

Finally we will examine the total entropy flow (TEF) of the storm. The TEF of a storm system is defined by summing the entropy flow over every grid point within a system at a constant pressure layer and then over every layer, leading TEF to be a single number standing for the intensity of entropy flow for the system at any given time. Entropy flow of a three-dimensional storm system through its boundary that is a curved surface $A$ can be worked out by integrating the divergence of the entropy flow vector $\vec{J}_{s}$ over the whole system volume $V$ (the summing over all the grid points encompassed by the curved surface is the discrete version of the integration) based on the well-known Gaussian formula [34]

$$
\iint_{\Sigma}(P \cos X+Q \cos Y+R \cos Z) d A=\iiint_{V}\left(\frac{\partial P}{\partial x}+\frac{\partial Q}{\partial y}+\frac{\partial R}{\partial z}\right) d x d y d z
$$

The integrand in the right hand side of equation 7 is just the divergence of the entropy flow vector $\vec{J}_{s}$ in this case.

In this paper the approximate total entropy flow is calculated on each pressure layer, by summing over all grid points within a circle centered on the storm's center with a radius of six degrees (thus the horizontal boundaries of the storm are defined), and then summed over all layers (thus the top and bottom pressure layer are treated as its vertical boundaries).

Figure 4. The evolution of total entropy flow (TEF) for TS Bilis in $6 \mathrm{~h}$ timesteps, with changes in the maximum surface wind velocity $\left(V_{\max }\right)$ as a reference.

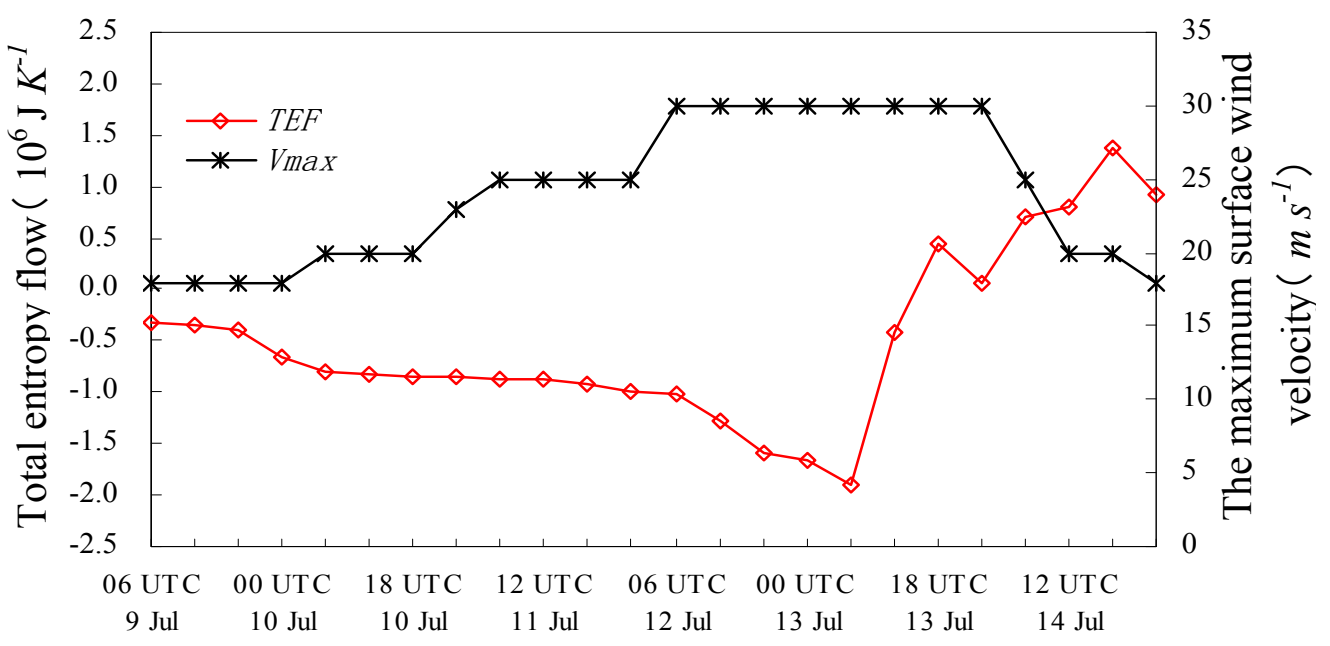

In view of that the evolution of the maximum surface wind of TS Bilis falls into three stages: (1) intensification before 0600 UTC 12 July during which the maximum wind monotonically increases from $18 \mathrm{~ms}^{-1}$; (2) maintenance from 0600 UTC 12 to 0000 UTC 14 July during which the maximum wind maintains its maximum of $30 \mathrm{~ms}^{-1}$, with a small drop in the central pressure from 980 to $975 \mathrm{hPa}$ at 0000 UTC 13 July; and, (3) decay from 0000 UTC 14 July during which the maximum wind continuously decreases from $30 \mathrm{~ms}^{-1}$, the calculations will then be made correspondingly to these stages (Figure 4). The results show that the total entropy flows around TS Bilis are negative and 
intensify monotonically during stage (1); in stage (2), where the maximum surface wind is kept at its maximum, the evolution of the TEF for the storm has a strong drop in the (negative) total entropy flow which then turns gradually to positive TEF; and, during stage (3) after 0000 UTC 14 July the total entropy flow around the storm and its neighborhood is positive. This implies that the growth of an atmospheric system may rely upon the negative entropy flow.

\section{Conclusion}

According to classical thermodynamics, the entropy of an isolated system will run to the maximum that corresponds to equilibrium in which no ordered structure forms. On the other hand, the nonlinear non-equilibrium thermodynamics points out that for an open system a negative entropy flow strong enough from its surroundings may drive the system to a state far from equilibrium so as to form dissipative structures, suggesting that negative entropy is something very important for structural creation and development in a thermodynamic system. However, the effect of entropy flow on the evolution of an atmospheric system is unknown.

This study reveals, via the entropy flow analysis of TS Bilis based on the entropy balance equation derived from the Gibbs relation and using NCEP/NCAR reanalysis data, that the entropy flow properties of this severe tropical storm are different in the different stages of its life-cycle and, especially that the growth of a severe atmospheric system may rely upon the negative entropy flow. As a result, entropy flow analysis might provide a powerful tool to understanding the mechanism responsible for the life cycle of an atmospheric system and associated weather events.

This is a preliminary case study and we intend to investigate a series of tropical storms and other vortices in the atmosphere. Only the generally accepted results reached in this field would potentially contribute to properly judging whether the entropy flow methodology can be generalized to the other relevant fields to investigate the life cycle of an ordered system, whether it is living or nonliving.

\section{Acknowledgements}

This work was jointly supported by the National Natural Science Foundation of China under Grants 40475022 and 40333028, and the Project of the Special Funds of Social Public Good for Scientific Research Institutions of China under Contract 2002DIA20013.

\section{Nomenclature}

$A$ System surface

$C_{p v}$ Specific heat at constant pressure for vapor

$\mathrm{m}^{2}$

$C_{p d}$ Specific heat at constant pressure for dry air

$\mathrm{m}^{2} \mathrm{~s}^{-2} \mathrm{~K}^{-1}$

$e$ Vapor pressure

$\mathrm{m}^{2} \mathrm{~s}^{-2} \mathrm{~K}^{-1}$

$g$ Gravitational acceleration

$\mathrm{m}^{-1} \mathrm{~kg} \mathrm{~s}^{-2}$

$k$ The $k_{t h}$ (chemical component)

$\vec{i}$ Unit vector at $\mathrm{x}$-direction

$\vec{J}_{k}$ Diffusive flow vector

$\mathrm{m} \mathrm{s}^{-2}$

$\vec{J}_{q}$ Heat flow vector

$\mathrm{m}^{-2} \mathrm{~kg} \mathrm{~s}^{-1}$

$\mathrm{kg} \mathrm{s}^{-3}$ 
$\vec{J}_{s}$ Entropy flow vector

$\mathrm{m}^{2} \mathrm{~kg} \mathrm{~s}^{-3} \mathrm{~K}^{-1}$

$\vec{j}$ Unit vector at y-direction

$\vec{k}$ Unit vector at z-direction

$m_{k}$ Relative molecular mass of component $k$

$N_{k}$ Fractional mass of component $k$

$N_{v}$ Fractional mass for vapor

$N_{d}$ Fractional mass for dry air

$P$ Component of a vector at $\mathrm{x}$-direction

$p$ Pressure

$\mathrm{m}^{-1} \mathrm{~kg} \mathrm{~s}^{-2}$

$Q$ Component of a vector at y-direction

$q$ Specific humidity

$R$ Component of a vector at z-direction in Cartesian coordinates

$R_{v}$ Gas constant for vapor

$\mathrm{m}^{2} \mathrm{~s}^{-2} \mathrm{~K}^{-1}$

$R_{d}$ Gas constant for dry air

$\mathrm{m}^{2} \mathrm{~s}^{-2} \mathrm{~K}^{-1}$

$r_{t h}$ The $r_{t h}$ (chemical reaction)

$S$ Component of a vector at z-direction

$s$ Specific entropy

$\mathrm{m}^{2} \mathrm{~s}^{-2} \mathrm{~K}^{-1}$

$T$ Temperature

$\mathrm{K}$

$u$ Velocity at $\mathrm{x}$-direction

$\mathrm{m} \mathrm{s}^{-1}$

$v$ Velocity at y-direction

$\mathrm{m} \mathrm{s}^{-1}$

$U$ Specific inner energy

$\mathrm{m}^{2} \mathrm{~s}^{-2}$

$V$ System volume

$\mathrm{m}^{3}$

$\vec{V}$ Velocity vector

$\mathrm{m} \mathrm{s}^{-1}$

$\vec{V}_{d}$ Velocity vector for dry air

$\mathrm{m} \mathrm{s}^{-1}$

$\vec{V}_{q}$ Velocity vector for vapor

$\mathrm{m} \mathrm{s}^{-1}$

$\vec{V}_{k}$ Velocity vector of component $k$

$\mathrm{m} \mathrm{s}^{-1}$

$x$ Cartesian coordinate at $\mathrm{x}$-direction

$\mathrm{m}$

$y$ Cartesian coordinate at $y$-direction

$\mathrm{m}$

$z$ Cartesian coordinate at $\mathrm{z}$-direction

$\mathrm{m}$

$X$ Angle between $\mathrm{x}$-axis and positive normal direction of system surface

$Y$ Angle between $y$-axis and positive normal direction of system surface

$Z$ Angle between z-axis and positive normal direction of system surface

\section{Greek}

$\alpha$ Specific volume

$\mu_{k}$ Chemical potential for component $k$

$\mathrm{m}^{3} \mathrm{~kg}^{-1}$

$\mathrm{m}^{2} \mathrm{~s}^{-2}$

$v_{k r} r_{t h}$ chemical reaction metrical coefficient of component $k$

$\rho$ Total density

$\mathrm{kg} \mathrm{m}^{-3}$

$\rho_{k}$ Density of component $k$

$\mathrm{kg} \mathrm{m}^{-3}$

$\rho_{v}$ Density of vapour

$\mathrm{kg} \mathrm{m}^{-3}$ 
$\rho_{d}$ Density of dry air

$\omega$ Vertical velocity in $p$-coordinates

$\omega_{r}$ Rate for the $r_{t h}$ chemical reaction $\mathrm{kg} \mathrm{m}^{-3}$

$\mathrm{mb} \mathrm{s}^{-1}$

$\mathrm{kg} \mathrm{m}^{-3} \mathrm{~s}^{-1}$

\section{References}

1. Ozawa, H.; Ohmura, A. Thermodynamics of a Global-Mean State of the Atmosphere-A State of Maximum Entropy Increase. J. Climate 1997, 10, 441-445.

2. Duane, G.S.; Curry, J.A. Entropy of a connecting water-air system and the interpretation of cloud morphogenesis. Q. J. R. Meteorol. Soc. 1997, 123, 605-629.

3. Barsugli, J.J.; Battisti, D.S. The basic effects of atmosphere-ocean thermal coupling on midlatitude variability. J. Atmos. Sci. 1998, 55, 477-493.

4. Egger, J. Numerical Generation of Entropies. Mon. Wea. Rev. 1999, 127, 2211-2216.

5. Nicolis, C. Irreversible thermodynamics of a simple atmospheric Model. Int. J. Bifurcation and Chaos 2002, 12, 2557-2566.

6. Liu, C.; Liu Y.; Kang, H. A new technique of physical dissipation and its application to a mesoscale numerical weather prediction model. Sci. China D 2002, 45, 769-780.

7. Liu, C.; Liu Y.; Xu, H. A physics-based diffusion scheme for numerical models. Geophys. Res. Lett. 2006, 33, L12805, doi:10.1029/2006GL025781.

8. Fraedrich, K.; Blender, R.; Scaling of Atmosphere and Ocean Temperature Correlations in Observations and Climate Models. Phys. Rev. Lett. 2003, 90, 108501 1-4.

9. Gade, H.G.; Gustafson, K. Application of classical thermodynamics principle to study of oceanic overturning circulation. Tellus 2004, 56, 371-387.

10. Liu, C.; Liu, Y. An attempt at improving a global spectral model by incorporating the second law of thermodynamics. Geophys. Res. Lett. 2005, 32, L03806, doi:10.1029/2004GL021602.

11. Iribarne, J.V.; Godson, W.L. Atmospheric Thermodynamics; D. Reidel Pub. Com: Boston, 1981; pp. 219-231.

12. Curry, J.A.; Webster, P.J. Thermodynamics of Atmospheres and Oceans; International Geophysics Series, Vol. 65, Academic Press: San Diego, CA, 1999; pp. 96-128.

13. Zdunkowski, W.; Bott, A. Thermodynamics of the Atmosphere; Cambridge University Press: Cambridge, UK, 2004; pp. 231-239.

14. Eady, E. T. Long waves and cyclone waves. Tellus 1949, 1, 33-52.

15. Prigogine, I. Thermodynamics of irreversible processes. Bull. Class. Sci. Acad. Roy. Belg. 1945, $31,600-621$.

16. De Groot, S.R.; Mazur, P. Non-equilibrium thermodynamics; North-Holland Publishing Company: Amsterdam, 1962; pp 410-425.

17. Schrödinger, E. What Is Life? Cambridge University Press (first published in 1944): Cambridge, U, 1992; pp. 19-25.

18. Olby, R. Schrödinger's problem: What is life? J. Hist. Biol. 1971, 4,119-148

19. Tsonis, A.A. Introduction to Atmospheric Thermodynamics; Cambridge University Press: Cambridge, UK, 2002; pp 69-72.

20. Peixoto, J.P. Entropy budget of the atmosphere. J. Geophys. Res. 1991, 96, 10981-10988. 
21. Paltridge, G.W. Global dynamics and climate - a system of minimum entropy exchange. $Q . J . R$. Meteorol. Soc. 1975, 101, 475-484.

22. Paltridge, G.W. Thermodynamic dissipation and the global climate system. Q. J. R. Meteorol. Soc. 1981, 107, 531-547.

23. Obrien, D.M. A yardstick for global entropy-flux. Q. J. R. Meteorol. Soc. 1997, 123, 243-260.

24. Stephens, G.L.; O'Brien, D.M. Entropy and climate I: ERBE observations of the entropy production of the earth. Q. J. R. Meteorol. Soc. 1993, 119, 121-152.

25. Stephens, G.L.; O’Brien, D.M. Entropy and climate II: Simple Models. Q. J. R. Meteorol. Soc. 1995, 121, 1773-1796.

26. Pauluis, O.; Balaji, V.; Held, I.M: Frictional dissipation in a precipitating atmosphere. J. Atmos. Sci., 2000, 57, 989-994.

27. Pauluis, O.; Held, I.M.: Entropy budget of an atmosphere in radiative-convective equilibrium. Part I: Maximum work and frictional dissipation. J. Atmos. Sci., 2002, 59, 140-149.

28. Mendes et al, On precursors of South American cyclogenesis. Tellus 2007, 59, 114-121.

29. Data available on https://dss.ucar.edu/datazone/dsszone/ds083.2/webgroups.html?grpidx=200607.

30 Prigogine, I. Introduction to Thermodynamics of Irreversible Processes; Charles C. Thomas: Springfield, IL, 1955; pp. 9-31

31. Onsager, L. Reciprocal relations in irreversible processes. I. Phys. Rev., 1931, 37, 405-426.

32. Mason, B. J. Physics of Clouds; Oxford University Press: London, 1971; pp. 119-123.

33. Liu, Y.; Zhang, D.L.; Yau, M.K. A multiscale numerical study of Hurricane Andrew (1992), Part I: Explicit simulation and verification. Mon. Wea. Rev. 1997, 125, 3073-3093.

34. Bronshtein, I.N.; Semendyayev, K.A. Handbook of mathematics, $3^{\text {rd }}$ Ed.; Springer-Verlag: Berlin, 1997; pp. 25-72.

(C) 2008 by the authors; licensee Molecular Diversity Preservation International, Basel, Switzerland. This article is an open-access article distributed under the terms and conditions of the Creative Commons Attribution license (http://creativecommons.org/licenses/by/3.0/). 